\title{
Amantadine in the prevention of clinical symptoms caused by SARS-CoV-2
}

\author{
Albert Cortés-Borra ${ }^{1}$ (i) $\cdot$ Gonzalo Emiliano Aranda-Abreu ${ }^{2}$ (i)
}

Received: 30 November 2020 / Revised: 2 February 2021 / Accepted: 4 February 2021 / Published online: 18 February 2021

(c) Maj Institute of Pharmacology Polish Academy of Sciences 2021

\begin{abstract}
Background Amantadine is a drug that can help in the prevention of SARS-CoV-2 symptomatology, as has been demonstrated in observational clinical studies.

Methods We searched in the PubMed database Clinical Studies of coronavirus-infected patients who have been treated with amantadine in a preventive manner as well as patients with Parkinson's disease.

Results Four clinical studies were found in which relatives of patients with COVID-19 had been prescribed the use of amantadine in a preventive manner to avoid the symptoms caused by the coronavirus.

Conclusion Amantadine is a drug that can be prescribed as a prophylactic that prevents symptomatology caused by SARSCoV-2 coronavirus.
\end{abstract}

Keywords Amantadine $\cdot$ SARS-CoV-2 $\cdot$ Prevention

\section{Introduction}

In December 2020, it will be 1 year since the SARS-CoV-2 viral disease was first reported. On March 11, 2020, the World Health Organization declared that this new disease caused by a coronavirus had been elevated from a public health problem to a pandemic [1]. Infections have occurred practically all over the world and the number of deaths is increasing every day. The efforts to find a drug that helps to decrease the clinical manifestations caused by the coronavirus have been practically constant.

Several medications have been used to decrease the clinical manifestations caused by the virus, such as hydroxychloroquine [2], ivermectin [3], dexamethasone [4], remdesivir [5].

Gonzalo Emiliano Aranda-Abreu

garanda@uv.mx

Albert Cortés-Borra

alcortesborra@gmail.com

1 Vall d'Hebron Barcelona Hospital Campus, Barcelona, Spain

2 Universidad Veracruzana/Instituto de Investigaciones Cerebrales, Xalapa, Veracruz, Mexico
Remdesivir and dexamethasone have been approved for the treatment of people with severe symptoms caused by the virus [6].

We have studied an adamantane derivative to treat the effects of SARS-CoV-2. Adamantane derivatives have been used as drugs with different biological activities. The best known are amantadine and memantine [7].

We have used a drug to treat the clinical symptoms caused by SARS-CoV-2 that has been approved by the FDA since 1976 for treatment of H1N1 influenza. Amantadine is a small molecule of low-molecular weight whose formula is $\mathrm{C}_{10} \mathrm{H}_{17} \mathrm{~N}$ and which is also used as an anti-Parkinsonic, since it seems to be involved in the release of dopamine from the nerve terminals [8]. Amantadine enters the $E$ channel of SARS-CoV-2 and prevents the virus to discharge its genetic content into the cell to generate new viral particles [9].

\section{Materials and methods}

A search was carried out on Pubmed of recent literature on studies conducted on patients treated with amantadine and that also the relatives who were in direct contact with the patients have been treated with this drug as a way to prevent the symptoms caused by COVID-19. We also searched for people with Parkinson's disease who are taking amantadine 
as part of their treatment, and who have been infected with SARS-CoV-2.

\section{Results}

\section{Amantadine in the prevention of SARS-CoV-2}

An observational study has shown that amantadine is an effective drug in the treatment of symptoms caused by SARS-CoV-2 [10].

It has been observed that patients with Parkinson's disease who were prescribed with amantadine as part of their treatment and were infected with the coronavirus did not develop the symptomatology caused by COVID-19 [11, 12].

In recent literature, there are few clinical cases related to the hypothetic role as preventive use of amantadine.

Aranda et al. studied a total of 15 patients of different ages who presented symptoms compatible with COVID-19. The average age of the patients studied was $48 \pm 16$ years. Ten of them were women and the rest were men. Four percent were suffering from hypertension and three percent from type 2 diabetes mellitus. Only one person reported cardiac disease. The main symptoms they reported were fever, dyspnea, headache, nausea and nasal congestion. Ten of the patients lost their sense of taste and smell. The treatment consisted of $100 \mathrm{mg}$ of amantadine, one tablet in the morning and one in the evening, for a period of 14 days. Most of them were prescribed $500 \mathrm{mg}$ of azithromycin, $200 \mathrm{mg}$ of celecoxib and $500 \mathrm{mg}$ of aspirin for a period of 6 days. Three patients had to receive nebulizations with ipratropium bromide/salbutamol, 3 times a day for 5 days. Two of the patients were administered oxygen (4Lpm) because their oxygen saturation was below $90 \%$. All patients recovered with amantadine treatment and none had to go to the hospital for mechanical ventilation. Family members who were in contact with them were prescribed amantadine $100 \mathrm{mg}$ twice daily as a prophylactic measure [10].

Cortés presents a 75-year-old woman with Parkinson's disease diagnosed 17 years previously is presented. Under medical supervision by her neurologist, the patient was receiving opicapone, $50 \mathrm{mg} /$ day, pramipexole, $2.1 \mathrm{mg} / \mathrm{day}$, levodopa, $1000 \mathrm{mg} /$ day, benserazide, $250 \mathrm{mg} /$ day and amantadine, $100 \mathrm{mg} /$ day and for hyperthyroidism levothyroxine, $25 \mathrm{mg} /$ day. Five years ago, the patient was diagnosed with gastric cancer treated surgically (Billroth II gastrectomy) and with chemotherapy before and after surgery, currently without recurrence of her cancer. After 7 days with a fever oscillating between 37.5 and $38.8{ }^{\circ} \mathrm{C}$ together with an unusual sporadic cough, mild diarrhea and fatigue, the patient's husband was diagnosed by PCR with COVID-19 which led to his hospital admission with a diagnosis of SARS-CoV-2 infection with bilateral pneumonia. Despite drug treatment and the administration of high-flow oxygen with a Monaghan mask, he died 5 days after admission. The female patient and wife of the above, did not present any symptoms of coronavirus infection: neither fever, nor cough, nor diarrhea, nor anosmia, despite having lived with her husband in a very intimate way, sharing a bed and exposed to the aerosols produced by her husband's persistent cough. Once her husband was admitted to hospital, she was isolated at the home of her daughter, who, after the death of her father, assumed the role of main caregiver. Both her daughter and her daughter's husband tested negative for COVID-19 by PCR [11].

Rejdak et al. studied 22 patients ( 10 with multiple sclerosis, 5 with Parkinson's disease and 7 with cognitive impairment) positive for SARS-CoV-2 and were receiving treatment with amantadine or memantine $(100 \mathrm{mg}$ q.d and $10 \mathrm{mg}$ b.i.d, respectively) for at least 3 months prior to exposure to infection. All patients were tested after reported personto-person contact with SARS-CoV-2 infected subjects and confirmed viral infection with SARS-CoV-2 PCR testing of upper and lower respiratory tract specimens. All of them were in quarantine for two weeks from the exposure documented, and none developed clinical manifestations of infectious disease. They also reported no significant change in neurological status in the course of primary nervous system disease [12].

Aranda et al. conducted a clinical study of a 57-year-old person who presented cold symptoms and body pain, with glucose levels of $200 \mathrm{mg} / \mathrm{dL}$, and was prescribed paracetamol (500 mg every $6 \mathrm{~h}$ ) and naproxen (550 mg daily for 5 days). The patient continued with his treatment for type 2 diabetes mellitus. Due to the persistent cough, azithromycin (500 mg) was added for a period of 3 days; however, the symptomatology continued, so it was decided to perform a real-time PCR test for SARS-CoV-2, which was positive. The treatment prescribed was $100 \mathrm{mg}$ of amantadine twice a day for 14 days. His asymptomatic relatives (wife and daughter) positive for SARS-CoV-2 were prescribed $100 \mathrm{mg}$ of amantadine twice daily as a preventive measure. The patient improved satisfactorily on the sixth day of treatment. The wife and daughter were symptom-free during the 14 days of treatment with amantadine [13].

Table 1 shows cases where amantadine has been used as a treatment and family members have been administered this drug as a measure to prevent them from getting symptoms from SARS-CoV-2 infection

Currently, there are a group of investigators who subscribe to the hypothesis that amantadine offers a protective effect to prevent the replication of the virus in patients who are taking it regularly.

Tipton and Wszolek from the Mayo Clinic suggest that "we must explore all possible options to prevent and seek treatment for COVID-19. The use of low-cost and readily available 
Table 1 Studies carried out with amantadine

\begin{tabular}{|c|c|c|}
\hline Clinical Studies & $\begin{array}{l}\text { Treatment } \\
\text { with amanta- } \\
\text { dine }\end{array}$ & Prevention of symptoms with amantadine \\
\hline 15 Patients infected with SARS-Cov-2 [10] & Yes & Family members received amantadine $100 \mathrm{mg}$ twice a day and had no symptoms \\
\hline Clinical case $[11]$ & Yes & SARS-CoV-2 infection, PCR positive did not present symptoms \\
\hline $\begin{array}{l}22 \text { Patients, } 10 \text { Multiple Sclerosis, } 5 \text { with } \\
\text { Parkinson's and } 7 \text { with Cognitive Impair- } \\
\text { ment [12] }\end{array}$ & Yes & SARS-CoV-2 infection, PCR positive did not present symptoms \\
\hline Clinical case [13] & Yes & $\begin{array}{l}\text { Wife and daughter SARS-CoV-2 PCR positive, took amantadine } 100 \mathrm{mg} \text { in the } \\
\text { morning and } 100 \text { in the evening, showed no symptoms }\end{array}$ \\
\hline
\end{tabular}

drugs is a low-risk and cost-effective approach. We propose amantadine and memantine as two possible candidates" [14]. Aranda-Abreu et al. demonstrate that amantadine blocks the viroporin channel of COVID-19, preventing the release of the viral nucleus in the cell cytoplasm and states that "the use of amantadine when the first symptoms of COVID-19 occur can mitigate the effects of the disease" [9].

In addition, Smieszek et al., conclude that "amantadine could be used as a potent agent to lower viral load if administered early enough in the course of COVID-19 infection and that the cumulation in lysosomes, if effective could reduce viral load, decrease organ spread within the host and decrease the severity and progression of associated disease." They suggest that more studies are needed to examine the role of amantadine [15].

Rejdak and Grieb in a study of patients with a range of neurological disorders concluded that "although their study had limitations due to the small sample size and cross-sectional approach, it indicated that the adamantanes amantadine or memantine exert a protective antiviral effect" and that, if confirmed, adamantanes could be useful in limiting SARS-CoV-2 infection and its clinical neurological sequelae" [12].

Cimolai expressed a view that, although not commonly discussed, the adamantanes should also be reevaluated at least in preliminary in vitro studies for various human coronaviruses [16]. Araujo et al. concluded that "In the absence of a vaccine or medication to help prevent or decrease the effects of the disease, we suggest that amantadine may reduce the effects of COVID-19" [17]. Wiwanitkit says there are many classic drugs that might be useful for management of COVID-19. Amantadine is an antiviral agent that is in that category. Its exact effect has to be further studied [18].

\section{Conclusion}

Amantadine due to its lipophilic nature can easily cross the biological membranes. Molecular docking studies have shown that amantadine can interact at the receptor binding domain of the S protein of SARS-CoV-2 [19].
In the case that the virus enters the cell, amantadine would interact with the E-channel, inhibiting the release of RNA into the cell.

This drug can be used preventively by personnel who are constantly exposed to the coronavirus, such as doctors, nurses, pharmacists, as well as people suffering from a disease, and the elderly can receive this drug as a protective measure in the sense that they can become infected with the coronavirus but not develop the characteristic symptoms of the disease.

Amantadine is well absorbed when administered orally and is also well tolerated by the digestive system. The recommended dose for an adult is one $100 \mathrm{mg}$ tablet twice daily for at least 14 days if the person is or has been exposed to SARS-CoV-2.

If the person does not have severe kidney failure, it may be taken indefinitely during the pandemic or if no vaccine is available. Amantadine has a moderate diuretic effect and is excreted unchanged in the urine via glomerular filtration.

In the Drug-bank database, it mentions that the reported lethal dose is $2 \mathrm{~g}$, the half-life is $10-14 \mathrm{~h}$.

In general, amantadine can be used in combination with other medications such as antihypertensives or antidiabetics [17].

At a time when the coronavirus has caused new outbreaks in some countries in Europe, the use of amantadine could prevent the development of symptoms of the disease in case an infection has occurred.

Author contributions Both authors designed and wrote the article.

\section{Compliance with ethical standards}

Conflict of interest No conflict of interest.

\section{References}

1. Cucinotta D, Vanelli M. WHO declares COVID-19 a pandemic. Acta bio-medica: AteneiParmensis. 2020;91(1):157-60. 
2. RECOVERY CG, Horby P, Mafham M, Linsell L, Bell JL, Staplin N. et al., Effect of hydroxychloroquine in hospitalized patients with Covid-19. N Engl J Med. 2020;383(21):2030-40.

3. Sharun K, Dhama K, Patel SK, Pathak M, Tiwari R, Singh $\mathrm{BR}$, et al. Ivermectin, a new candidate therapeutic against SARS-CoV-2/COVID-19. Ann ClinMicrobiolAntimicrob. 2020;19(1):23.

4. Ledford H. Coronavirus breakthrough: dexamethasone is first drug shown to save lives. Nature. 2020;582(7813):469.

5. Choi SW, Shin JS, Park SJ, Jung E, Park YG, Lee J. et al., Antiviral activity and safety of remdesivir against SARS-CoV-2 infection in human pluripotent stem cell-derived cardiomyocytes. Antiviral Res. 2020; p. 104955.

6. Kim A, Gandhi R. Coronavirus disease 2019 (COVID-19): management in hospitalized adults. UpToDate. 2020.

7. Dembitsky VM, Gloriozova TA, Poroikov VV. Pharmacological profile of natural and synthetic compounds with rigid adamantane-based scaffolds as potential agents for the treatment of neurodegenerative diseases. BiochemBiophys Res Commun. 2020;529(4):1225-41.

8. McKimm-Breschkin JL, Fry AM. Meeting report: 4th ISIRV antiviral group conference: novel antiviral therapies for influenza and other respiratory viruses. Antivir Res. 2016;129:21-38.

9. Abreu GEA, Aguilar MEH, Covarrubias DH, Durán FR. Amantadine as a drug to mitigate the effects of COVID-19. Med Hypotheses. 2020;140:109755.

10. Aranda-Abreu GE, Aranda-Martínez JD, Araújo R, HernándezAguilar ME, Herrera-Covarrubias D, Rojas-Durán F. Observational study of people infected with SARS-Cov-2, treated with amantadine. Pharmacol Rep. 2020;72(6):1538-41.
11. Cortés BA. Does amantadine have a protective effect against COVID-19. NeurolNeurochir Pol. 2020;54(3):284-5.

12. Rejdak K, Grieb P. Adamantanes might be protective from COVID-19 in patients with neurological diseases: multiple sclerosis, parkinsonism and cognitive impairment. MultSclerRelatDisord. 2020;42:102163.

13. Aranda-Abreu GE, Aranda-Martínez JD, Araújo R. Use of amantadine in a patient with SARS-CoV-2. J Med Virol. 2020.

14. Tipton PW, Wszolek ZK. What can Parkinson's disease teach us about COVID-19. NeurolNeurochir Pol. 2020;54(2):204-6.

15. Smieszek SP, Przychodzen BP, Polymeropoulos MH. Amantadine disrupts lysosomal gene expression: a hypothesis for COVID19 treatment. Int J Antimicrob Agents. 2020;55(6):106004.

16. Cimolai N. Potentially repurposing adamantanes for COVID-19. J Med Virol. 2020;92(6):531-2.

17. Araújo R, Aranda-Martínez JD, Aranda-Abreu GE. Amantadine treatment for people with COVID-19. Arch Med Res. 2020;51(7):739-40.

18. Wiwanitkit V. Amantadine, COVID-19 and Parkinsonism. Arch Med Res. 2020;51(7):714.

19. Baig AM, Khaleeq A, Syeda H. Docking prediction of amantadine in the receptor binding domain of spike protein of SARS-CoV-2. ACS PharmacolTransiSci. 2020;3(6):1430-3.

Publisher's Note Springer Nature remains neutral with regard to jurisdictional claims in published maps and institutional affiliations. 